\title{
Infección por Clostridium difficile: descripción de las cepas NAP1/027 y de otros serotipos en un centro de alta complejidad de Cali, Colombia, 2012-2015
}

\author{
José Millán Oñate-Gutiérrez ${ }^{1,2,3}$, Janier Segura ${ }^{1}$, Adriana Correa $^{4}$, Erika Cantor ${ }^{5}$, María \\ Virginia Villegas ${ }^{1}$ \\ ${ }^{1}$ Servicio de Medicina Interna, Centro Médico Imbanaco, Cali, Colombia \\ ${ }^{2}$ Resistencia bacteriana, CIDEIM, Cali, Colombia \\ ${ }^{3}$ Departamento de Medicina Interna, Escuela de Medicina, Universidad del Valle, Cali, Colombia \\ ${ }^{4}$ Servicio de Dermatología, Centro Médico Imbanaco, Cali, Colombia \\ ${ }^{5}$ Instituto de Investigación, Centro Médico Imbanaco, Cali, Colombia
}

Introducción. Clostridium difficile ocasiona infecciones hospitalarias que resultan en altas tasas de morbilidad y mortalidad. La cepa NAP1/027 se ha asociado con una mayor producción de toxinas y con una mayor gravedad, lo que aumenta la carga de la enfermedad. Objetivo. Describir la epidemiología de las infecciones asociadas con $C$. difficile y las características de la cepa NAP1/027.

Materiales y métodos. Se hizo un estudio observacional basado en la revisión de las historias clínicas de los pacientes con muestras de heces positivas para $C$. difficile identificadas mediante la prueba Xpert ${ }^{\mathrm{TM}}$ entre el 2012 y el 2015 en un hospital de alta complejidad. La gravedad de la enfermedad se evaluó con el índice ATLAS.

Resultados. Se incluyeron 42 casos de pacientes infectados, 9 de los cuales fueron positivos para la cepa NAP1/027. El uso de antibióticos antes de la infección durante más de siete días fue más frecuente en los casos de pacientes con muestras negativas para NAP1/027. En la mitad de los pacientes, la duración de la diarrea fue mayor de cinco días y no hubo diferencias según el tipo de cepa $(p>0,05)$. Los casos de pacientes positivos para la cepa NAP1/027 se caracterizaron por presentar deposiciones fétidas y sanguinolentas. La gravedad de la infección fue similar entre los grupos.

Recibido: 30/06/17 Aceptado: $22 / 08 / 18$ Publicado: $27 / 08 / 18$

Citación:

Oñate-Gutiérrez JM, Segura J, Correa A, Cantor E, Villegas MV. Infección por Clostridium difficile: descripción de las cepas NAP1/027 y de otros serotipos en un centro de alta complejidad de Cali, Colombia, 2012-2015. Biomédica. 2019;39(Supl.1):63-70

https://doi.org/10.7705/biomedica.v39i2.3950

Correspondencia:

José Millán Oñate-Gutiérrez, Servicio de Medicina Interna, Centro Médico Imbanaco, Calle $5 \mathrm{C} \mathrm{N}^{\circ} 39$ 33, primer piso, Cali, Colombia

Teléfono: (572) 682 1000, extensión 14091; fax: (572) 5146015

jose.onate@imbanaco.com.co y millanonate@gmail. com

Contribución de los autores:

Jose Millán Oñate-Gutiérrez: participación en todas las etapas del estudio

Erika Cantor: creación y análisis de la base de datos Janier Segura y María Virginia Villegas: recolección de los datos

Todos los autores participaron en el diseño del estudio, la revisión de la literatura y la redacción del manuscrito.

\section{Financiación:}

No se requirió financiación para el presente estudio, el cual se llevó a cabo en las instalaciones del

Centro Médico Imbanaco de Cali, S.A.

Conflicto de intereses:

Los autores declaran no tener conflicto de intereses.
Conclusión. Se comprobó la circulación de la cepa NAP1/027, pero su presencia no supuso diferencias clínicas significativas con respecto a otras cepas, lo cual podría deberse al limitado número de pacientes en este estudio. Sin embargo, su presencia debe alertar a los médicos y a las instituciones de salud, dada su frecuente asociación con la gravedad de la infección y la mortalidad.

Palabras clave: Clostridium difficile; diarrea; antibacterianos; infecciones bacterianas/ epidemiología; enterocolitis seudomembranosa.

Clostridium difficile infection: Description of NAP1/027 and non NAP1/027 strains in a high complexity center in Cali, Colombia, 2012-2015

Introduction: Clostridium difficile causes nosocomial infections leading to high morbidity and mortality. The NAP1/027 strain is associated with a higher toxin production and disease severity, which increases the load of the disease.

Objective: To describe the epidemiology of the infections associated with $C$. difficile and the characteristics related to the NAP1/027 strain.

Materials and methods: This was an observational study based on the revision of clinical registries of patients with fecal samples that were positive for $C$. difficile identified by the Xpert test ${ }^{\mathrm{TM}}$ between 2012 and 2015 in a high complexity institution. The severity of the disease was evaluated by means of the ATLAS score.

Results: We included 42 infected cases, 9 of which were positive for the NAP1/027strain. The use of antibiotics previous to the infection for more than seven days was more frequent in patients with negative results for NAP1/027. The duration of diarrhea in half of the patients was longer than five days and there were no differences according to the type of strain ( $p>0.05$ ). Positive cases for the NAP1/027 strain were characterized by presenting fetid and bloody stools. The severity of the infection was similar between the groups. Conclusions: In Colombia, the NAP1/027 strain circulates without significant clinical differences, which could be due to the limited number of patients. Nevertheless, the existence of NAP1/027 should alert physicians and health institutions because of its high association with severity and mortality.

Keywords: Clostridium difficile; diarrhea; anti-bacterial agents; bacterial infections/ epidemiology; enterocolitis, pseudomembranous. 
Clostridum difficile es un microorganismo de creciente relevancia que actualmente se considera el agente causal más importante de las infecciones hospitalarias en los Estados Unidos (1). El espectro de las infecciones ocasionadas por $C$. difficile incluye desde las asintomáticas hasta aquellas con megacolon tóxico y colitis fulminante, complicaciones presentes en 3 a $8 \%$ de los pacientes infectados (2-5). Estas infecciones se asocian con un incremento de los costos de hospitalización, así como con recurrencia y aumento de la mortalidad (4-6).

Recientemente, se reportó la aparición de nuevos serotipos de $C$. difficile y de una cepa hipervirulenta, la NAP1/027 (North American PFGE type1/ ribotype 027), que se asocia con una mayor producción de toxinas y, por consiguiente, con una mayor gravedad de las manifestaciones clínicas (7-9)

En Colombia, ya se ha informado de la presencia de esta cepa hipervirulenta $(10,11)$, pero hay pocos datos sobre la epidemiología hospitalaria y el papel del agente patógeno en los hospitales. Varios autores han descrito factores asociados con la presencia de $C$. difficile en el país (1013) y, previamente, se había informado sobre una prevalencia de 10 casos por cada 10.000 pacientes por año en el Centro Médico Imbanaco (10).

Los Centers for Disease Control and Prevention (CDC) recomiendan que los hospitales y clínicas deben incluir el diagnóstico de $C$. difficile en la evaluación de los casos indicativos de esta infección, además de establecer políticas de vigilancia local y de detección de los factores de riesgo asociados con esta infección y con su gravedad (14).

En este contexto, el objetivo del presente trabajo fue describir la epidemiología de las infecciones asociadas con $C$. difficile en un hospital de alta complejidad, así como la presentación clínica observada, la gravedad de los casos y las características relacionadas con la presencia de la cepa NAP1/027.

\section{Materiales y métodos}

\section{Diseño del estudio}

Se llevó a cabo un estudio observacional basado en la revisión de las historias clínicas de los pacientes con muestras de heces positivas para $C$. difficile entre el 2012 y el 2015 en un hospital de alta complejidad. Para su identificación, se utilizó la prueba Xpert C. difficile ${ }^{\mathrm{TM}}$ (Cepheid, Sunnyvale, CA, EE. UU.) y su sistema GeneXpert'TM. Esta técnica permite la detección de los genes de $C$. difficile con una sensibilidad mayor del $90 \%$ y, además, identifica el ribotipo responsable de la infección.

Se definió como caso de diarrea asociada con $C$. difficile el de aquellos pacientes con deposiciones diarreicas y un resultado positivo en la reacción en cadena de la polimerasa (PCR) para este microorganismo. La recurrencia se definió como la presencia de dos episodios de diarrea en un tiempo no mayor de ocho semanas. El caso adquirido en la comunidad se definió como el de aquellos pacientes que no presentaban antecedentes de hospitalización en el último mes antes de que presentaran alguna sintomatología.

\section{Muestra de estudio}

Durante el período del estudio, se encontraron 48 muestras de heces positivas para $C$. difficile. En el análisis se excluyeron 6 casos en cuyas historias clínicas institucionales no se informaba la presencia de la infección. 
Con el sistema GeneXpert ${ }^{\mathrm{TM}}$, se determinó la presencia de $C$. difficile y del ribotipo 027. Los 42 pacientes restantes se clasificaron en dos grupos según la presencia o la ausencia del ribotipo NAP1/027, y se hicieron comparaciones entre ellos.

\section{Recolección de la información}

Se revisaron las historias clínicas de cada paciente y se recolectó la información de la presencia de comorbilidades (diabetes mellitus, enfermedad hematológica-oncológica, insuficiencia renal crónica, enfermedad cardiopulmonar o trasplantes, entre otras). Asimismo, se analizaron los signos vitales al iniciarse los síntomas y se registraron los valores de los leucocitos, la creatinina y la proteína $C$ reactiva en el momento del diagnóstico. Además, se analizaron la duración de la administración y el tipo de antibióticos utilizados antes del inicio de la diarrea, y se caracterizaron las deposiciones descritas por los pacientes.

La gravedad de la enfermedad se calificó con el puntaje ATLAS (Antimicrobial Testing Leadership And Surveillance) $(15,16)$

\section{Análisis estadístico}

La información se analizó con el programa estadístico Stata 13.0 ${ }^{\mathrm{TM}}$. La descripción de las características de los casos positivos o negativos para la cepa NAP1/027, se hizo con estadísticas de tendencia central (media, mediana) y de dispersión (desviación estándar y rango intercuartílico) en el caso de las variables continuas, y con distribuciones de frecuencias relativas y absolutas para las variables categóricas.

En la comparación de las variables categóricas entre los casos positivos y negativos para la cepa NAP1/0.27, se utilizó la prueba de ji al cuadrado, o prueba de Fisher, y para las cualitativas, la prueba t de Student o la U de Mann-Whitney, según los criterios de normalidad. El nivel de significación se fijó en 0,05 .

\section{Consideraciones éticas}

Este estudio fue aprobado por el Comité de Ética en Investigación del Centro Médico Imbanaco.

\section{Resultados}

Durante el período de estudio, se encontraron 42 casos de infección por C. difficile en las historias clínicas del Centro. En 9 (21,4\%) de las muestras positivas para $C$. difficile, se identificó la cepa NAP1/027 mediante PCR.

En el cuadro 1, se presentan las características generales y clínicas de los pacientes. Al comparar los dos grupos con cepas de $C$. difficile, con presencia o ausencia del ribotipo NAP1/027, no se observaron diferencias en la edad; la mitad de los casos de $C$. difficile se presentó en adultos mayores de 53 años, así como en pacientes con leucemia o falla renal, y en aquellos bajo tratamiento por otras infecciones. Ocho $(19,0 \%)$ de los casos positivos para $C$. difficile provenían de la comunidad y, de estos, tres eran positivos para la cepa NAP1/027, es decir, casi la tercera parte.

La administración de antibióticos por más de siete días antes de la infección fue más frecuente entre los casos por cepas sin el serotipo NAP1/027 $(p<0,05)$, con una mediana de 11 días $(R I Q=8-16)$, en comparación 
con la mediana de 7 días $(\mathrm{RIQ}=6-11)$ en aquellos con $C$. difficile de serotipo NAP1/027. Los carbapenémicos fueron los antibióticos más utilizados antes del inicio de la diarrea, y se emplearon únicamente en los casos positivos para $C$. difficile, mas no para aquellos con la cepa NAP1/027. Los antiácidos (inhibidores de la bomba de protones) se utilizaron en siete de cada diez casos positivos para $C$. difficile, con una proporción similar entre los infectados con la cepa NAP1/027 y aquellos con otros serotipos (cuadro 1).

Cuadro 1. Características generales de los pacientes

\begin{tabular}{|c|c|c|c|c|}
\hline Variables & $\begin{array}{c}\text { Universo } \\
(\mathrm{N}=42)\end{array}$ & $\begin{array}{l}\text { Positivos para } \\
\text { NAP1/027 }(n=9)\end{array}$ & $\begin{array}{l}\text { Negativos para } \\
\text { NAP1/027 }(n=33)\end{array}$ & $\mathbf{p}$ \\
\hline Edad (años) [mediana (RIC)] & $53,0(22,5-64,0)$ & $48,0(20,5-59,5)$ & $55,0(21,0-67,5)$ & 0,589 \\
\hline $\begin{array}{l}\text { Sexo }[\mathrm{n}(\%)] \\
\text { Masculino } \\
\text { Femenino }\end{array}$ & $\begin{array}{l}15(35,7) \\
27(64,3)\end{array}$ & $\begin{array}{l}2(22,2) \\
7(77,8)\end{array}$ & $\begin{array}{l}13(39,4) \\
20(60,6)\end{array}$ & 0,451 \\
\hline $\begin{array}{l}\text { Signos vitales } \\
\text { Presión arterial sistólica }(\mathrm{mm} \mathrm{Hg})[\text { media } \pm \mathrm{DE}] \\
\text { Presión arterial diastólica }(\mathrm{mm} \mathrm{Hg})[\text { media } \pm \mathrm{DE}] \\
\text { Frecuencia cardiaca (latidos por minuto) [mediana }(\mathrm{RIC})] \\
\left.\text { Temperatura }\left({ }^{\circ} \mathrm{C}\right) \text { [mediana }(\mathrm{RIC})\right]\end{array}$ & $\begin{array}{r}115,6 \pm 17,1 \\
67,8 \pm 11,3 \\
90,0(83,5-108,2) \\
36,5(36,1-37,3)\end{array}$ & $\begin{array}{r}113,0 \pm 12,5 \\
67,6 \pm 11,8 \\
92,0(85,5-107,5) \\
36,5(36,1-37,1)\end{array}$ & $\begin{array}{r}116,6 \pm 18,3 \\
67,6 \pm 11,3 \\
90,0(81,5-112,5) \\
36,5(36,0-37,4)\end{array}$ & $\begin{array}{l}0,607 \\
0,781 \\
0,747 \\
0,154\end{array}$ \\
\hline $\begin{array}{l}\text { Exámenes de laboratorio } \\
\text { Leucocitos (células/ml) [mediana (RIC)] } \\
\text { Creatinina (mg/dl) [mediana (RIC)] } \\
\text { PCR [mediana (RIC)] }\end{array}$ & $\begin{array}{r}10,3(3,1-15,8) \\
0,66(0,48-1,31) \\
66,7(23,5-145,7)\end{array}$ & $\begin{array}{r}11,5(9,2-25,9) \\
0,58(0,52-1,07) \\
75,6(23,6-156,2)\end{array}$ & $\begin{array}{r}10,1(2,3-15,6) \\
0,67(0,45-1,41) \\
37,2(12,4-95,2)\end{array}$ & $\begin{array}{l}0,154 \\
0,276 \\
0,473\end{array}$ \\
\hline $\begin{array}{l}\text { Diagnósticos [n (\%)] } \\
\text { Anemia } \\
\text { Falla renal } \\
\text { Leucemias } \\
\text { Infección urinaria } \\
\text { Otra infección } \\
\text { Cáncer } \\
\text { Hipotiroidismo } \\
\text { Enfermedad coronaria } \\
\text { Hipertensión arterial sistémica } \\
\text { Diabetes }\end{array}$ & $\begin{array}{r}4(9,5) \\
9(21,4) \\
11(26,2) \\
10(23,8) \\
10(23,8) \\
8(19,0) \\
4(9,5) \\
6(14,3) \\
9(21,4) \\
6(14,3)\end{array}$ & $\begin{array}{l}2(22,2) \\
2(22,2) \\
2(22,2) \\
1(11,1) \\
4(44,1) \\
2(22,2) \\
3(33,3) \\
2(22,2) \\
1(11,1) \\
0(0,0)\end{array}$ & $\begin{array}{r}2(6,1) \\
7(21,2) \\
9(27,3) \\
9(27,3) \\
6(18,2) \\
6(18,2) \\
1(3,0) \\
4(12,1) \\
8(24,2) \\
6(18,2)\end{array}$ & $\begin{array}{l}0,196 \\
0,999 \\
0,999 \\
0,416 \\
0,181 \\
0,999 \\
0,026 \\
0,593 \\
0,655 \\
0,312\end{array}$ \\
\hline $\begin{array}{l}\text { Cirugías previas } \\
\text { Sí } \\
\text { No }\end{array}$ & $\begin{array}{l}23(54,8) \\
19(45,2)\end{array}$ & $\begin{array}{l}4(44,4) \\
5(55,6)\end{array}$ & $\begin{array}{r}19(57,6) \\
414(42,4)\end{array}$ & 0,707 \\
\hline $\begin{array}{l}\text { Antibióticos previos [n (\%)] } \\
\text { Sí }\end{array}$ & $40(95,2)$ & $9(100,0)$ & $31(93,9)$ & 0,999 \\
\hline $\begin{array}{l}\text { Duración de los antibióticos (días) [n (\%)] } \\
\leq 7 \\
>7\end{array}$ & $\begin{array}{l}10(25,6) \\
29(74,4)\end{array}$ & $\begin{array}{l}5(55,6) \\
4(44,4)\end{array}$ & $\begin{array}{r}5(16,7) \\
25(83,3)\end{array}$ & 0,032 \\
\hline $\begin{array}{l}\text { Tipo de antibiótico [n (\%)] } \\
\text { Linezolida } \\
\text { Clindamicina } \\
\text { Cefepime } \\
\text { Fluoroquinolona } \\
\text { Clindamicina } \\
\text { Vancomicina } \\
\text { Cefalosporina, } 1 \mathrm{~g} \\
\text { Cefalosporina, } 2 \mathrm{~g} \\
\text { Cefalosporina, } 3 \mathrm{~g} \\
\text { Carbapenémicos } \\
\text { Penicilinas } \\
\text { Penicilina inhibidores de la betalactamasa }\end{array}$ & $\begin{array}{r}3(7,7) \\
3(7,7) \\
9(23,1) \\
9(23,1) \\
3(7,7) \\
6(15,4) \\
1(2,6) \\
3(7,7) \\
3(7,7) \\
11(28,2) \\
5(12,8) \\
8(20,5)\end{array}$ & $\begin{array}{r}0(0,0) \\
1(11,1) \\
1(11,1) \\
2(22,2) \\
1(11,1) \\
1(11,1) \\
1(11,1) \\
1(11,1) \\
2(22,2) \\
0(0,0) \\
1(11,1) \\
3(33,3)\end{array}$ & $\begin{array}{r}3(10,0) \\
2(6,7) \\
8(26,7) \\
7(23,3) \\
2(6,7) \\
5(16,7) \\
0(0,0) \\
2(6,7) \\
1(3,3) \\
11(36,7) \\
4(13,3) \\
5(16,7)\end{array}$ & $\begin{array}{l}0,999 \\
0,556 \\
0,654 \\
0,999 \\
0,556 \\
0,999 \\
0,231 \\
0,556 \\
0,127 \\
0,040 \\
0,999 \\
0,355\end{array}$ \\
\hline $\begin{array}{l}\text { Antiácidos [n (\%)] } \\
\text { Sí }\end{array}$ & $31(73,8)$ & $7(77,8)$ & $24(72,7)$ & 0,999 \\
\hline
\end{tabular}

RIC: rango intercuartílico; DE: desviación estándar 
En la mitad de los pacientes, la diarrea persistió durante más de cinco días, sin diferencias en cuanto al tipo de cepa $(p>0,05)$. Los pacientes con infección por la cepa de serotipo NAP1/027, se caracterizaron por presentar deposiciones fétidas $(77,8 \%)$ y sanguinolentas $(44,4 \%)$, en comparación con aquellos con otros serotipos, en los cuales dichas proporciones fueron de 30,3 y $15,1 \%$, respectivamente

La gravedad de la infección fue similar entre los casos con la cepa NAP1/027 y aquellos con $C$. difficile de otros serotipos. La tercera parte de las infecciones por $C$. difficile se consideraron graves según el puntaje ATLAS $(15,16)$. En un caso negativo para la cepa NAP1/027, fue necesario hacer una hemicolectomía para controlar la infección. Siete $(16,7 \%)$ de los pacientes fallecieron durante la estancia hospitalaria. Tres $(7,1 \%)$ de las muertes se atribuyeron a la infección por $C$. difficile, pero ninguna cepa era del serotipo NAP1/027 en estos casos (cuadro 2).

Cuadro 2. Características de la deposición y gravedad de la enfermedad

\begin{tabular}{|c|c|c|c|c|c|c|c|}
\hline \multirow{2}{*}{$\begin{array}{l}\text { Variables } \\
\text { Diarrea (días) [mediana (RIC)] }\end{array}$} & \multicolumn{2}{|c|}{$\begin{array}{l}\text { Universo } \\
(\mathrm{N}=42)\end{array}$} & \multicolumn{2}{|c|}{$\begin{array}{l}\text { Positivos para } \\
\text { NAP1/027 }(n=9)\end{array}$} & \multicolumn{2}{|c|}{$\begin{array}{l}\text { Negativos para } \\
\text { NAP1/027 }(n=33)\end{array}$} & \multirow{2}{*}{$\begin{array}{c}\mathbf{p} \\
0,516\end{array}$} \\
\hline & 5 & $(3-9)$ & 4 & $(1-8)$ & 5 & $(3-9)$ & \\
\hline \multicolumn{8}{|l|}{ Heces } \\
\hline Fétidas & 17 & $(40,5)$ & 7 & $(77,8)$ & 10 & $(30,3)$ & 0,019 \\
\hline Sanguinolentas & 9 & $(21,4)$ & 4 & $(44,4)$ & 5 & $(15,1)$ & 0,080 \\
\hline Con moco & 7 & $(16,7)$ & 1 & $(11,1)$ & 6 & $(18,2)$ & 0,999 \\
\hline \multicolumn{8}{|l|}{ Calificación según ATLAS } \\
\hline Leve & 26 & $(61,9)$ & 6 & $(66,7)$ & 20 & $(60,6)$ & 0,999 \\
\hline Grave & 16 & $(38,1)$ & 3 & $(33,3)$ & 13) & $(39,4)$ & \\
\hline \multicolumn{8}{|l|}{ Recidiva } \\
\hline Sí & 2 & $(4,8)$ & 1 & $(11,1)$ & 1 & $(3,0)$ & 0,387 \\
\hline \multicolumn{8}{|l|}{ Complicaciones } \\
\hline Sepsis & 5 & $(11,9)$ & 0 & $(0,0)$ & 5 & $(15,1)$ & 0,567 \\
\hline Muerte & 3 & $(7,1)$ & 0 & $(0,0)$ & 3 & $(9,1)$ & 0,999 \\
\hline Muerte por cualquier causa & 7 & $(16,7)$ & 0 & $(0,0)$ & 7 & $(21,2)$ & 0,314 \\
\hline \multicolumn{8}{|l|}{ Estancia en UCI [n (\%)] } \\
\hline Sí & 14 & $(33,3)$ & 2 & $(22,2)$ & 12 & $(36,4)$ & 0,692 \\
\hline \multicolumn{8}{|l|}{ Estancia hospitalaria (días) } \\
\hline [mediana $(\mathrm{RIC})]$ & 18,5 & $(6,0-41,0)$ & 7,0 & $4,0-93,0)$ & 21,0 & $(7,0-41,0)$ & 0,373 \\
\hline
\end{tabular}

RIC: rango intercuartílico; UCl: unidad de cuidados intensivos

Catorce (33,3\%) de los 42 pacientes con infección por C. difficile requirieron hospitalización en la unidad de cuidados intensivos, sin diferencias según el tipo de cepa. En dos de infectados con la cepa de serotipo NAP1/027, el tratamiento se cambió posteriormente a vancomicina. Por otra parte, solo un caso negativo para esta cepa requirió este medicamento. La infección fue recurrente en dos (4,8\%) de los 42 pacientes positivos para $C$. difficile, uno de ellos con el serotipo NAP1/027 y que había recibido ampicilina sulbactam como profiláctico antes de una colecistectomía; el otro fue negativo para la cepa NAP1/027 y recibió tratamiento con vancomicina por infección en una prótesis de rodilla (cuadro 2).

\section{Discusión}

Clostridum difficile es el principal agente patógeno responsable de infecciones hospitalarias en los Estados Unidos (1). La morbimortalidad asociada con este germen se ha incrementado en los últimos años, probablemente por la aparición de una cepa extremadamente virulenta (7). Sin embargo, algunos 
autores han planteado que la mortalidad asociada con $C$. difficile depende del estado clínico y de la inmunosupresión del paciente $(4,5)$. En este estudio, la mortalidad asociada con $C$. difficile fue del $7,1 \%$, comparable con las registradas en otras series de casos con tasas entre el 6 y el $10 \%(6,8)$.

Los antibióticos carbapenémicos se emplearon únicamente en los casos negativos para la cepa hipervirulenta, hallazgo que debe constatarse en estudios con un número mayor de pacientes y en diferentes centros para establecer su causalidad. En el presente estudio, debe tenerse en cuenta que la mayoría de los pacientes que recibieron carbapenémicos estaban hospitalizados y que, en el hospital de estudio, entre las cepas circulantes no predominan las virulentas.

El puntaje del ATLAS es una herramienta clínica validada para diferenciar entre los casos graves, y los leves o moderados $(15,16)$. En ella, se utilizan variables clínicas y solo un indicador de laboratorio, la albúmina, para diferenciarlos según la presentación clínica inicial. Es importante establecer la gravedad del paciente en el momento del diagnóstico, para administrar un tratamiento antimicrobiano adecuado que, en los casos graves, se recomienda sea con vancomicina oral o con fidaxomicina (14). Solo 16 (38\%) de los 42 pacientes se calificaron como graves en el momento del diagnóstico y no hubo diferencias entre los dos grupos (con ausencia 0 presencia del serotipo NAP1/027), según la gravedad inicial, la permanencia en la unidad de cuidados intensivos o los días de hospitalización, lo que coincide con otros estudios en los que no se ha observado una mayor virulencia en los infectados por la cepa NAP1/027 (4).

Por otra parte, el porcentaje de pacientes con infecciones originadas en la comunidad fue de $19 \%$, hallazgo que concuerda con lo reportado previamente en la ciudad (10). Esto plantea la necesidad de una política de uso regulado de los medicamentos antimicrobianos en el ámbito ambulatorio y de restricción de la venta de antibióticos sin fórmula médica.

La mayoría de los pacientes recibió tratamiento con metronidazol, y las tasas de mortalidad y recurrencia no se asociaron con el antibiótico empleado. Sin embargo, el uso de metronidazol se ha asociado con recurrencias, especialmente en aquellos casos cuya presentación clínica inicial fue grave (17), diferencias que pueden atribuirse al tamaño de la muestra, por lo que se requiere un estudio con mayor número de casos, preferentemente multicéntrico, para corroborar estos hallazgos.

La tasa de recurrencias fue del $4,8 \%$, inferior a lo reportado previamente en otros estudios (4) y aunque dicha tasa fue baja, se asoció con el uso continuado de antibióticos para controlar otras infecciones, lo que aumenta el daño colateral sobre la microbiota y favorece la reaparición de $C$. difficile. Solo un paciente requirió hemicolectomía para controlar su infección; en este sentido, debe considerarse el uso de otras opciones terapéuticas para este tipo de situaciones de difícil tratamiento y control, por ejemplo, los anticuerpos monoclonales, la fidaxomicina (18) o la tigeciclina intravenosa (19).

Dado que este estudio se hizo en un solo hospital, no es posible extrapolar los datos a nivel nacional, aunque sus resultados ponen de manifiesto la necesidad de hacer un estudio nacional multicéntrico para evaluar el impacto de la cepa NAP1/027. Los pocos casos positivos para la cepa NAP1/027 constituyen un punto de partida para determinar posibles factores relacionados con la aparición de esta nueva cepa. 
Es importante conocer la realidad epidemiológica de $C$. difficile en el país, con el fin de adoptar medidas que limiten su diseminación en los hospitales, y favorezcan el diagnóstico y el tratamiento temprano y efectivo.

\section{Agradecimientos}

Al Servicio de Laboratorio y al Instituto de Investigación del Centro Médico Imbanaco por su apoyo durante la realización de este proyecto.

\section{Referencias}

1. Maggil S, Edwards J, Stat M, Bamberg W, Beldavs Z, Dumyati G, et al. Multistate pointprevalence survey of health care-associated infections. N Engl J Med. 2014;370:1198-208. https://doi.org/10.1056/NEJMoa1306801

2. Leffler D, Lamont T. Clostridium difficile infections. N Engl J Med. 2015;372:1539-48. https://doi.org/10.1056/NEJMra1403772

3. Zea JW, Salazar CL. Enfermedad asociada a Clostridium difficile: prevalencia y diagnóstico por laboratorio. Infectio. 2012;16:211-22. https://doi.org/10.1016/S0123-9392(12)70016-4

4. Depestel DD, Aronoff DM. Epidemiology of Clostridium difficile infection. J Pharm Pract. 2013;26:464-75. https://doi.org/10.1177/0897190013499521

5. Rodríguez-Pardo D, Almirante B, Bartolome RM, Pomar V, Mirelis B, Navarro F, et al. Epidemiology of Clostridium difficile infection and risk factors for unfavorable clinical outcomes: Results of a hospital-based study in Barcelona, Spain. J Clin Microbiol. 2013;51:1465-73. https://doi.org/10.1128/JCM.03352-12

6. Magee G, Strauss ME, Thomas SM, Brown H, Baumer D, Broderick KC. Impact of Clostridium difficile-associated diarrhea on acute care length of stay, hospital costs, and readmission: A multicenter retrospective study of inpatients, 2009-2011. Am J Infect Control. 2015;43:1148-53. https://doi.org/10.1016/j.ajic.2015.06.004

7. See I, Mu Y, Cohen J, Beldavs ZG, Winston LG, Dumyati G, et al. NAP1 strain type predicts outcomes from Clostridium difficile infection. Clin Infect Dis. 2014;58:1394-400. https://doi.org/10.1093/cid/ciu125

8. Morfin-Otero R, Garza-González E, Aguirre-Díaz SA, Escobedo-Sánchez R, EsparzaAhumada S, Pérez-Gómez HR, et al. Clostridium difficile outbreak caused by NAP1/BI/027 strain and non-027 strains in a Mexican hospital. Braz J Infect Dis. 2016;20:8-13. https://doi.org/10.1016/j.bjid.2015.09.008

9. Marsh JW, Arora R, Schlackman JL, Shutt KA, Curry SR, Harrison LH. Association of relapse of Clostridium difficile disease with BI/NAP1/027. J Clin Microbiol. 2012;50:4078-82. https://doi.org/10.1128/JCM.02291-12

10. Oñate-Gutiérrez J, Villegas M, Correa A. Prevalencia y factores relacionados con la infección por Clostridium difficile en un centro hospitalario de alta complejidad en Cali (Colombia). Infectio. 2017;21:9-14. https://doi.org/10.1016/j.infect.2015.12.002

11. Gualtero S, Abril LA, Camelo N, Sánchez SD, Silva E, Dávila FA, et al. Características de la infección por Clostridium difficile en una institución de alta complejidad y reporte de la circulación en Colombia de la cepa hipervirulenta NAP17027. Biomédica. 2017;37:7-12. https://doi.org/10.7705/biomedica.v37i4.3244

12. Otero-Regino W, González A, Gómez-Zuleta M. Prevalencia de diferentes tipos de colitis en personas adultas mayores. Revista Colombiana de Gastroenterología. 2009;24:272-8.

13. Becerra MG, Ospina S, Atehortúa SL, Berbesi DY. Factores de riesgo para la infección por Clostridium difficile. Infectio. 2011;15:220-6.

14. Cohen S, Gerding D, Johnson S, Kelly C, Loo V, McDonald L, et al. Clinical practice guidelines for Clostridium difficile infection in adults: 2010 update by the society for healthcare epidemiology of America (SHEA) and the infectious diseases society of America (IDSA). Infect Control Hosp Epidemiol. 2010;31:431-55. https://doi.org/10.1086/651706

15. Hernández-García R, Garza-González E, Miller M, Arteaga-Muller G, Galván-de los Santos AM, Camacho-Ortiz A. Application of the ATLAS score for evaluating the severity of Clostridium difficile infection in teaching hospitals in México. Braz J Infect Dis. 2015;19:399402. https://doi.org/10.1016/j.bjid.2015.05.005 
16. Miller MA, Louie T, Mullane K, Weiss K, Lentnek A, Golan Y, et al. Derivation and validation of a simple clinical bedside score (ATLAS) for Clostridium difficile infection which predicts response to therapy. BMC Infect Dis. 2013;13:148. https://doi.org/10.1186/1471-2334-13-148

17. Di X, Bai N, Zhang X, Liu B, Ni W, Wang J,et al. A meta-analysis of metronidazole and vancomycin for the treatment of Clostridium difficile Infection, stratified by disease severity. Braz J Infect Dis. 2015;19:339-49. https://doi.org/10.1016/j.bjid.2015.03.006

18. Debast S, Bauer M, Kuijper E. European Society of Clinical Microbiology and Infectious Diseases: Update of the treatment guidance document for Clostridium difficile infection. Clin Microbiol Infect. 2014;20(Suppl.2):1-26. https://doi.org/10.1111/1469-0691.12418

19. Mathur H, Rea MC, Cotter PD, Ross RP, Hill C. The potential for emerging therapeutic options for Clostridium difficile infection. Gut Microbes. 2014;5:696-710. https://doi.org/10.4161/19490976.2014.983768 